Relations industrielles

Industrial Relations

\title{
McRae, Susan, Part-Time Work in the European Union: The Gender Dimension
}

\section{Francine Mayer}

Volume 51, numéro 3, 1996

URI : https://id.erudit.org/iderudit/051123ar

DOI : https://doi.org/10.7202/051123ar

Aller au sommaire du numéro

Éditeur(s)

Département des relations industrielles de l'Université Laval

ISSN

0034-379X (imprimé)

1703-8138 (numérique)

Découvrir la revue

Citer ce compte rendu

Mayer, F. (1996). Compte rendu de [McRae, Susan, Part-Time Work in the European Union: The Gender Dimension]. Relations industrielles / Industrial Relations, 51(3), 600-602. https://doi.org/10.7202/051123ar

Tous droits réservés (C Département des relations industrielles de l'Université Laval, 1996
Ce document est protégé par la loi sur le droit d'auteur. L’utilisation des services d'Érudit (y compris la reproduction) est assujettie à sa politique d'utilisation que vous pouvez consulter en ligne.

https://apropos.erudit.org/fr/usagers/politique-dutilisation/ 
le droit au travail n'est plus, comme il a pu l'être, le "droit opérateur du droit international des droits économiques de la personne " (p. 452). D'ailleurs, en droit interne, le droit du travail n'est-il pas en passe de devenir, plus extensivement et souplement, un "droit de l'activité "? (G. Lyon-Caen, Le droit du travail, une technique réversible, Paris, Dalloz, 1995, p. 6.) Le Pacte sur les droits économiques prend ainsi un relief particulier. Mais, ne pourrait-on pas parler davantage de complémentarité entre les instruments de l'ONU et ceux de l'OIT, ces derniers présentant l'avantage de la spécificité et de la proximité des milieux de l'activité dite d'emploi ? Une telle complémentarité irait d'ailleurs dans le sens de la thèse de l'interdépendance générale des droits économiques de la personne sur le plan international.

L'ouvrage, dont on aura voulu simplement évoquer l'objet, représente une contribution scientifique majeure à l'étude du rôle du droit international des droits économiques de la personne en ce qu'il en constitue une synthèse élaborée des éléments constitutifs et en propose vigoureusement, encore une fois, une orientation qui tienne compte de l'exclusion sociale qui atteint les sociétés occidentales.

PIERRE VERGE

Université Laval

\section{Part-time Work in the European Union: The Gender Dimension \\ par Susan MCRAE, Luxembourg, Office for Official Publications of the European Communities, 1995, 73p., ISBN 92-827-3975-9.}

Dans cette monographie de 73 pages, Susan McRae présente les résultats d'une enquête menée en 1989-1990 par la Fondation européenne pour l'amélioration des conditions de vie et de travail dans huit pays européens (ex-Allemagne de l'Ouest, Belgique, Danemark, Espagne, Irlande, Italie, Pays-Bas et Royaume-Uni). Il s'agit d'une enquête en deux parties, l'une effectuée auprès des employeurs de 3520 établissements du secteur privé de 10 salariés ou plus, l'autre menée, quand cela était possible, auprès des représentants des travailleurs de ces établissements (1 621). Cette étude vise à apporter un nouvel éclairage sur la pertinence et les conséquences du travail à temps partiel pour les femmes européennes, en identifiant ses avantages et ses inconvénients. L'exclusion du champ de l'enquête des entreprises de moins de 10 salariés et des entreprises du secteur public, qui offrent généralement plus d'avantages aux salariés à temps partiel, exige évidemment d'interpréter les résultats de façon nuancée. Par ailleurs, bien qu'elle reconnaisse l'existence de différences nationales, l'auteure retient une perspective européenne et présente généralement les résultats de l'enquête pour les huit pays couverts pris comme une entité homogène.

L'auteure rappelle d'abord quelques faits sur le travail à temps partiel en Europe. Le temps partiel (TP) concerne essentiellement les femmes et peut être un moyen de concilier travail rémunéré et vie familiale ou autres activités. Le TP n'est pas toujours choisi. Même s'il est volontaire, il peut être désavantageux pour les femmes si les horaires de travail conviennent peu à leurs besoins, s'il entraîne des risques de déqualification professionnelle et des difficultés de retour au temps complet, ou si la législation du travail ne garantit pas des salaires et avantages sociaux au prorata des heures travaillées. Le TP peut toutefois réduire les risques de discontinuités d'emploi pour les femmes qui ont de jeunes enfants.

Pour atteindre les objectifs visés sans disposer d'une enquête menée directement auprès des salariées, l'auteure partage l'échantillon des établissements couverts par l'enquête en trois groupes de tailles approximativement égales, selon 
le " coefficient de représentation féminine " (mesuré comme le rapport entre la proportion de femmes qui travaillent dans l'établissement et la proportion de femmes dans la population active du pays concerné) : les établissements à forte représentation féminine, les établissements à faible représentation féminine, et les établissements qu'on peut classer entre ces deux extrêmes. L'auteure est d'avis que la ségrégation selon le sexe observée dans les lieux de travail peut affecter les expériences professionnelles des femmes. De plus, parce qu'elle estime que les raisons pour lesquelles les employeurs introduisent le TP dans leur établissement peuvent aussi avoir des conséquences sur les expériences de travail à TP vécues par les femmes, l'auteure distingue deux types d'établissements, selon la stratégie en matière de temps partiel : (i) les établissements de type stratégie d'entreprise, où le TP est introduit principalement pour des raisons économiques ou organisationnelles, et (ii) les établissements de type stratégie individuelle, où les possibilités de TP existent principalement en réponse à des demandes de réduction du temps de travail de la part des salariés. Les résultats de l'enquête sont donc présentés en fonction des variables "coefficient de réprésentation féminine" et "stratégie en matière de temps partiel ".

L'auteure décrit ensuite les caractéristiques des établissements selon la stratégie adoptée en matière de TP et présente les opinions des employeurs et des représentants des travailleurs quant aux avantages et aux inconvénients du travail à TP. Dans les établissements à stratégie d'entreprise, le marché du travail externe est la principale source de recrutement des travailleurs à TP. Ces établissements ont davantage tendance à connaitre de fortes variations dans le volume de travail au cours d'une journée ou d'une journée à l'autre que les établissements à stratégie individuelle. Leurs effectifs se composent donc d'une plus forte proportion de salariés à TP.
Par ailleurs, la direction et les représentants des travailleurs de ces établissements estiment généralement que le TP présente de nombreux avantages pour l'entreprise, notamment en termes de compétitivité et de qualité des produits et services offerts. C'est une image contrastée que l'on observe dans les établissements à stratégie individuelle, où les travailleurs à TP sont surtout recrutés au sein de l'entreprise, parmi les salariés à temps complet qui ont exprimé le souhait de réduire leurs heures de travail, et où la direction estime généralement que le travail à TP apporte peu d'avantages à l'entreprise. Dans ces établissements, les représentants des travailleurs sont plus souvent favorables au TP et ont tendance à considérer qu'il améliore les perspectives professionnelles des femmes. D'ailleurs, les salariées à TP risquent moins que leurs collègues des établissements à stratégie d'entreprise de se voir offrir, pour un travail identique, un salaire horaire inférieur ou des avantages sociaux proportionnellement moindres que les salariées à temps complet. Elles sont aussi moins susceptibles d'être soumises à des horaires de travail irréguliers ou à l'insécurité d'emploi, mais ne sont pas plus favorisées que leurs collègues des établissements à stratégie d'entreprise en termes de perspectives de promotion.

L'auteure aborde finalement la question de la mobilité entre les emplois à temps complet et les emplois à temps partiel. Elle constate que la stratégie en matière de TP affecte cette mobilité. D'abord, la probabilité que la direction d'une entreprise reçoive des demandes de réduction du temps de travail de la part des salariées à temps complet est plus élevée dans les établissements de type stratégie individuelle, notamment quand le coefficient de représentation féminine est grand. Le taux de réponses favorables aux demandes de temps réduit est cependant moins sensible à la stratégie en matière de $\mathrm{TP}$, notamment dans les entreprises à forte représentation féminine, qu'à la «culture professionnelle 
de l'organisation ", c'est-à-dire l'attitude qu'ont les dirigeants quant à la capacité des femmes ayant de jeunes enfants d'ētre de bons travailleurs à TP. Une attitude négative va de pair avec des possibilités réduites de passage vers le TP. Dans les établissements à faible représentation féminine, une attitude négative de la direction est aussi liée, pour un travail identique, à des salaires horaires inférieurs et des avantages sociaux proportionnellement moins importants pour les salariées à TP comparativement à leurs collègues à temps complet et à des perspectives de formation limitées. Par ailleurs, les dirigeants des établissements de type stratégie individuelle répondent plus souvent favorablement aux demandes de retour vers le temps complet que leurs collègues des établissements à stratégie d'entreprise, où les postes de travail sont souvent organisés sur une base de temps partiel, pour répondre aux exigences de flexibilité de l'entreprise plus qu'aux besoins des salariées. Le coefficient de représentation féminine agit aussi positivement sur la probabilité que l'employeur accueille favorablement les demandes de retour au temps complet.

L'auteure conclut en soulignant que les femmes qui choisissent de travailler à TP ne désirent évidemment pas des emplois précaires, mal rémunérés, leur offrant des perspectives professionnelles limitées, et que les États devraient prioritairement s'assurer que le développement des emplois à temps partiel ne conduise pas à accroître le nombre de mauvais emplois.

Voilà brièvement exposés les principaux sujets abordés par l'auteure. Son étude est intéressante parce qu'elle présente des résultats tirés d'une large enquête qui n'avait pas encore été exploitée. On peut regretter que l'auteure ne situe pas son étude dans la littérature, qu'elle dépasse peu le niveau descriptif et ne s'aventure que timidement dans l'analyse des résultats. À cet égard, il aurait été utile de fournir en annexe le questionnaire de l'enquête, car la formulation exacte des questions est importante pour bien interpréter les données. Mais au-delà de ces réserves, parce qu'elle élargit nos connaissances sur le rôle du lieu de travail sur l'expérience de travail à temps partiel des femmes, l'auteure atteint les objectifs qui ont motivé son étude.

FRANCINE MAYER

Université du Québec à Montréal

\section{Gender and Racial Inequality at Work: The Sources and Consequences of Job Segregation}

by Donald TOMASKOVIC-DEVEY, Cornell Studies in Industrial and Labour Relations, Ithaca, NY, ILR Press, 1993, ISBN 0-87546-304-5 and ISBN 0-87546-305-3 (pbk).

This study, which uses the 1989 survey of employed adults in North Carolina, focuses on the following questions:

1. Why do men and women rarely work in the same jobs in the same workplaces?

2. Why do the majority of white Americans work in jobs filled only by others of their own racial group?

3. Can the supply side explanations that focus on individual skills and the de- mand side explanations that focus on job and organizational power provide adequate light on the subject?

The thesis of this study of segregation by gender and race is that jobs can be profoundly influenced by their sex and racial composition. The author argues that jobs have gender. There are black jobs. There are white male jobs and these jobs often develop certain advantages in the workplace over and above the skill levels or even the organizational power of the people who hold them. 